\title{
A quantitative risk assessment approach for mosquito-borne diseases: malaria re-emergence in southern France Nicolas Ponçon ${ }^{1}$, Annelise Tran ${ }^{2,3}$, Céline Toty ${ }^{1}$, Adrian JF Luty ${ }^{4}$ and Didier Fontenille*1
}

\author{
Address: ${ }^{1}$ Institut de recherche pour le développement, UR016, 911 avenue Agropolis, BP64501, 34394, Montpellier, cedex 5, France, ${ }^{2}$ Centre de \\ coopération internationale de la recherche agronomique pour le développement, UPR22, campus de Baillarguet, 34398, Montpellier, cedex 5, \\ France, ${ }^{3}$ UMR TETIS, Maison de la Télédétection, 500 rue JF Breton, 34093, Montpellier, cedex 5, France and ${ }^{4}$ Medical Microbiology, Radboud \\ University Nijmegen Medical Centre, Nijmegen, The Netherlands \\ Email: Nicolas Ponçon - nicolas.poncon@agriculture.gouv.fr; Annelise Tran - annelise.tran@cirad.fr; Céline Toty - toty@mpl.ird.fr; \\ Adrian JF Luty - a.luty@mmb.umcn.nl; Didier Fontenille* - fontenil@mpl.ird.fr \\ * Corresponding author
}

Published: I August 2008

Malaria Journal 2008, 7:147 doi:10.1186/1475-2875-7-147
Received: 12 February 2008

Accepted: I August 2008

This article is available from: http://www.malariajournal.com/content/7/I//47

(c) 2008 Ponçon et al; licensee BioMed Central Ltd.

This is an Open Access article distributed under the terms of the Creative Commons Attribution License (http://creativecommons.org/licenses/by/2.0), which permits unrestricted use, distribution, and reproduction in any medium, provided the original work is properly cited.

\begin{abstract}
Background: The Camargue region is a former malaria endemic area, where potential Anopheles vectors are still abundant. Considering the importation of Plasmodium due to the high number of imported malaria cases in France, the aim of this article was to make some predictions regarding the risk of malaria re-emergence in the Camargue.

Methods: Receptivity (vectorial capacity) and infectivity (vector susceptibility) were inferred using an innovative probabilistic approach and considering both Plasmodium falciparum and Plasmodium vivax. Each parameter of receptivity (human biting rate, anthropophily, length of trophogonic cycle, survival rate, length of sporogonic cycle) and infectivity were estimated based on field survey, bibliographic data and expert knowledge and fitted with probability distributions taking into account the variability and the uncertainty of the estimation. Spatial and temporal variations of the parameters were determined using environmental factors derived from satellite imagery, meteorological data and entomological field data. The entomological risk (receptivity/infectivity) was calculated using 10,000 different randomly selected sets of values extracted from the probability distributions. The result was mapped in the Camargue area. Finally, vulnerability (number of malaria imported cases) was inferred using data collected in regional hospitals.
\end{abstract}

Results: The entomological risk presented large spatial, temporal and Plasmodium speciesdependent variations. The sensitivity analysis showed that susceptibility, survival rate and human biting rate were the three most influential parameters for entomological risk. Assessment of vulnerability showed that among the imported cases in the region, only very few were imported in at-risk areas.

Conclusion: The current risk of malaria re-emergence seems negligible due to the very low number of imported Plasmodium. This model demonstrated its efficiency for mosquito-borne diseases risk assessment. 


\section{Background}

In the past, malaria was endemic and constituted a major health issue in France in marshy areas, particularly the Camargue, which was an active focus until the beginning of the $20^{\text {th }}$ century. Malaria decreased drastically due to the draining of marshes, rearing of livestock, improvement of housing and living conditions and the use of quinine [1]. Malaria disappeared from the Camargue after World War II: the last Plasmodium vivax malaria epidemic occurred in 1943, with about 400 estimated cases [2]. Recent entomological surveys reported huge Anopheles populations in this area [3-5], and considered Anopheles (Anopheles) hyrcanus as being the main potential malarial vector based on its anthropophilic feeding behaviour and abundance $[4,6]$. Thus, the Camargue is currently facing an "anophelism without malaria" situation. Moreover, autochthonous transmission was recently suspected in the French Mediterranean coast in 2006 [7], supporting the idea that southern France remains suitable for malaria transmission. The number of imported malaria cases have increased dramatically since the 1970s, in parallel with increased international travels, with an average of about 6,400 cases per year for the last ten years in France, leading to a massive Plasmodium introduction from endemic countries into France $[8,9]$. These observations suggest that the malaria situation needs to be re-examined, and the aim of this paper is to infer current risk of malaria reemergence, to identify hot spots for malaria re-emergence in the Camargue and to develop a generic model for mosquito transmitted diseases.

The risk of malaria re-emergence in an area (i.e., the recurrence of malaria transmission in an area) may be estimated by three factors: receptivity, infectivity and vulnerability [10-13], usually assessed at the regional scale in a semi-qualitative way (Figure 1,2) [14]. In this article, a quantitative entomological risk, which is the product of receptivity and infectivity, is calculated and the impact of vulnerability is discussed. The main objective of this work was to estimate the risk of malaria re-emergence at the local scale, considering the temporal and spatial local variations of the three components in order to identify hot spots for malaria resurgence in the Camargue.

The main difficulty that occurs when modeling is the quantification of biological parameters, especially for entomological data, as the field and laboratory studies are very painstaking, time-consuming and only rarely permit conclusions on a precise value. In this article, a probabilistic approach, taking into account the uncertainties and variability of inputs, was applied to a vector-borne disease, which constitutes an innovative method. Even if the malaria situation needs to be re-examined in the Camargue, this disease does not constitute a major health issue. The aim of this article is not to provide a public health tool that can be used to control malaria in the

Receptivity is expressed by the vectorial capacity index and is calculated following the mathematical formula: $m a^{2} p^{n /-I n p ~(~} m, a, p, n$ are described in the Figure 2). It refers to the expected quantum of new inoculations of man per infective malaria case per day that the mosquito population would distribute to man considering that all vector females biting the malaria case become infected and transmit.

Infectivity (b) is the degree of susceptibility of an Anopheles species (or population) to different Plasmodium species (or strains).

Vulnerability is the number of malaria cases or, more precisely, gametocyte carriers, imported in the area.

Entomological risk is defined, in this article, as the product of receptivity and infectivity (receptivity*infectivity). Hence, it refers to the expected quantum of new inoculations of man per infective malaria case per day that the mosquito population would distribute to man.

Malariogenic potential is the risk estimate of malaria re-emergence and is determined by three factors: receptivity, infectivity and vulnerability. It is usually assessed in a semi-qualitative and relative way.

Ro (Basic reproductive rate) is the total number of malaria cases derived from one infective case that the mosquito population would distribute to man in the absence of immunity. It is the product of the vectorial capacity, the susceptibility and the human recovery rate $(1 / r)$, and is calculated with the following mathematical formula: $\left(m a^{2} p^{n /-\ln p)}{ }^{*} b^{*} 1 / r\right.$, where $1 / r$ is the length in days during which humans are infectious. R0 must equal at least 1 for disease persistence and increase. For values less than 1 , the disease is supposed to decrease.

\section{Figure I}

Risk of malaria re-emergence. 
$\boldsymbol{m}$ is the vector-host ratio, i.e., the anopheline density in relation to man.

$a$ is the vector biting rate and is calculated as follows: $a=h / u$. It refers to the average number of people bitten by one mosquito in one day.

ma is the human biting rate, i.e., the number of bites per human per day.

$\boldsymbol{h}$ is the proportion of blood meals taken from people (as opposed to other animals that are not infected with human malaria). It is also named anthropophily.

$\boldsymbol{u}$ is the length in days of the trophogonic cycle, considering the approximation that An. hyrcanus takes only one blood meal per cycle. The trophogonic cycle is the period delimited by two consecutive blood meals, and comprised of the time necessary for blood digestion, to find a pool to lay eggs in, and to find a host for the next blood meal.

$\boldsymbol{p}$ is the daily survival rate, i.e., the probability of a mosquito surviving through one whole day.

$\boldsymbol{n}$ is the length in days of the sporogonic development, i.e. the time necessary for parasites to fulfil a complete development from ingested gametocytes during the blood meal to sporozoites in the salivary glands (the stage at which the parasite is transmissible to humans).

$\boldsymbol{b}$ is the susceptibility of mosquitoes to Plasmodium species, i.e. their intrinsic ability to replicate and transmit Plasmodium. It expresses the proportion of female mosquitoes developing parasites after an infective blood meal.

\section{Figure 2}

\section{Entomological factors.}

Camargue, but to present an innovative approach to spatialized quantitative risk assessment applied to a vectorborne disease.

Receptivity and infectivity were estimated for the potential vector An. hyrcanus as i) it is now considered as the main potential malaria vector and ii) other Anopheles species are rare in the Camargue area. However, the approach developed here is applicable to other mosquito species.

\section{Materials, methods and data processing Study area}

The Camargue is the main wetland area in Southern France and covers the Rhone river delta. This area has a Mediterranean climate characterized by warm, dry summers and mild, wet winters. Total annual rainfall usually ranges between 500 and $700 \mathrm{~mm}$ and occurs mainly in autumn, and the annual mean temperature is $14^{\circ} \mathrm{C}$.

Water pools and marshes cover a large part of the Camargue. Water is provided either by rains or a very tight canal network diverted from the river Rhone used to irrigate paddies or to fill marshes. Management of water is at the level of individual field owners depending on use: grazing for horses, cows or sheep, exploitation of reeds or rice, hunting reserves for waterfowl and nature preservation. Landscapes in the Camargue are strongly affected by the duration of submersion and the salinity of the soils.
They are organized roughly in a south-north gradient of salinity, with agricultural land and reed marshes in the north and natural salty ponds and salt marshes in the south [15].

Moreover, there are various forms of agriculture (including vineyard, paddies, market gardening, fruit growing and exploitation of reeds) and rice, which covers more than 18,000 hectares in the Camargue, is the main cultivation [16]. Livestock includes horses, cows and sheep.

The Camargue hosts nearly 100,000 permanent inhabitants distributed between towns, hamlets and isolated houses. Moreover, the number of people increases in summer due to tourism.

\section{Quantitative risk assessment using a probabilistic approach}

The objective of this method was to organize and analyse scientific information in order to infer the risk of malaria re-emergence taking into account the variability and uncertainty of the input components and the final risk estimate. Such analysis, using reiterated simulations, have been performed for a decade for risk assessment in food microbiology, for example $[17,18]$. Information and data for the development of the entomological risk model were obtained from field surveys, literature, unpublished data and expert opinion. Biological parameters were estimated 
Beta distribution describes the true probability of an event occurring, given $x$ trials and $s$ successes. It is expressed as follows: probability (event) = Beta $\left(\alpha_{1}, \alpha_{2}\right)$, where $\alpha_{1}=s+1$ and $\alpha_{2}=x-s+1$. We used this when the process of biological data collection or biological experiment could be assimilated as a number of $s$ successes among $x$ trials, which was the case for the parity rate estimate ( $s$ parous females among $x$ dissected females) and susceptibility estimate ( $s$ positive mosquitoes among $x$ tested).

Pert distribution is generated from the Beta distribution, and requires three parameters: the minimum value $(v)$, the most likely value $(w)$ and the maximum value $(z)$. Pert $(v, w, z)$ means that $v$ is the minimum value of the distribution, $z$ the maximum, and $w$ the most likely. It is used to model expert knowledge in the absence of an extended dataset allowing one to determine the distribution of biological parameters. In this case, experts are asked to provide $v, w$ and $z$.

\section{Figure 3}

Probability distributions.

by probability distributions in a plausible way that is coherent and conceivable and they were fitted with Pert or beta distribution $[18,19]$ (Figure 3). An amount of 10,000 reiterated simulations generated by the Latin Hypercube method associated with the probability distributions was used to describe both variability and uncertainty within the input parameters and the model $[20,21]$. The outcome is a statistical distribution of risk, as well as a mean value of the risk estimate. Sensitivity analysis was performed to point out factors responsible for the main impact on the risk estimate. The @ risk $^{\circledast}$ (Palisade Corporation) software version $4,5,3$ was used.

\section{Meteorological data}

Daily temperatures (2005) and mean monthly temperatures (from 2002 to 2006) recorded by MeteoFrance at Aigues-Mortes (western Camargue) and at Tour du Vallat (south-eastern Camargue) were used. Daily temperatures were smoothed with a moving average (running mean) of three days to filter daily variations. For both types of data (daily and monthly), we calculated the average temperature for the Camargue based on the two stations. Humidity was recorded in 2005 at Marais du Vigueirat. These meteorological data were used to estimate some of the biological parameters of An. hyrcanus.

\section{Entomological data: receptivity}

In order to assess receptivity, it was necessary to evaluate the human biting rate (ma), the vector biting rate $(a)$, the survival rate $(p)$ and the sporogonic cycle $(n)$ and their spatial and temporal variations (Figure 2 ).

\section{Space and time-dependency}

Although we sampled a huge amount of An. hyrcanus (125,848 specimens captured, 504 females dissected), it has not been possible to estimate precisely potential spatial variations of some biological parameters. Thus and due to the small size of the Camargue, the biology of $A n$. hyrcanus was considered to be homogeneous in the whole area, which means that the vector biting rate $(a)$, survival rate $(p)$ and sporogonic cycle $(n)$ (Figure 2 ) did not vary spatially. On the contrary, as An. hyrcanus presence and density depend on the biotopes and the season $[4,5]$, the vector-host ratio $m$ (and hence the human biting rate $(m a)$ ) (Figure 2) presents a strong spatial heterogeneity (Table 1). Spatial variations were assessed based on a Geo-

Table I: Space, time and Plasmodium species-dependent variations in receptivity, infectivity and vulnerability.

\begin{tabular}{|c|c|c|c|c|}
\hline & \multirow[b]{2}{*}{ Components } & \multicolumn{3}{|c|}{ Variation } \\
\hline & & $\begin{array}{c}\text { Space } \\
\text { dependent }\end{array}$ & $\begin{array}{c}\text { Time } \\
\text { dependent }\end{array}$ & $\begin{array}{c}\text { Plasmodium-species } \\
\text { dependent }\end{array}$ \\
\hline \multirow{6}{*}{ Receptivity } & m (vector-host ratio) & YES & YES & NO \\
\hline & a (vector-biting rate) & $\mathrm{NO}$ & YES & $\mathrm{NO}$ \\
\hline & h (anthropophily) & NO & NO & NO \\
\hline & u (length of the trophogonic cycle) & NO & YES & $\mathrm{NO}$ \\
\hline & $\mathrm{P}$ (survival rate) & NO & NO & $\mathrm{NO}$ \\
\hline & n (sporogonic cycle) & $\mathrm{NO}$ & YES & YES \\
\hline Infectivity & b (susceptibility) & $\mathrm{NO}$ & $\mathrm{NO}$ & YES \\
\hline Vulnerability & & YES & YES & YES \\
\hline
\end{tabular}


graphic Information System (GIS) computing data for each 30 meter-wide pixel in the Camargue [22].

All parameters were considered time-dependent, except the Anopheles anthropophily (h) and, as a practical approximation, the survival rate (p) (Table 1). Time-variations, which are detailed below, were described at a monthly time step.

\section{Human biting rate (ma)}

The presence and density of An. hyrcanus were inferredusing remote sensing, entomological adults and larvae collections [22]. Analysis of larval data led to the definition of a larval index that was calculated for each pixel in the Camargue based on environmental key factors. An adult abundance index was generated from the larval index and was also calculated for each pixel in the study area. Comparison of the adult abundance index and the maximum number of An. hyrcanus captured in the same pixel with CDC-light traps $+\mathrm{CO}_{2}$ showed a highly significant linear regression, allowing us to infer, using key environmental factors, the maximum number of An. hyrcanus captured during the year with $\mathrm{CDC}$-light traps $+\mathrm{CO}_{2}$ for each pixel in the Camargue [22].

The mean annual dynamics of An. hyrcanus in the Camargue was inferred from the results of several capture campaigns conducted during several years, in several places, using several capture methods (Table 2). Specimens of this species were collected from March to October, and presented huge abundance variation (none of them were collected during winter). In each capture month, the mean percentage of collected mosquitoes (among the total number of mosquitoes captured during the year) were $0,0,0,4[1 ; 7], 12[2 ; 21], 66[51 ; 80], 18$ [9; 27], 0 from March to October, respectively (with the associated confidence interval in brackets), and the maximum number of An. hyrcanus captured during the year refers to the month of August. Hence, the spatial distribution of the annual maximum number of An. hyrcanus was combined with the mean annual dynamics in order to determine, for each pixel and for each month in the Camargue, the number of An. hyrcanus captured with CDC-light traps $+\mathrm{CO}_{2}$.

From this, the human biting rate $(m a)$ was inferred from a comparison between mean CDC-light trap captures and mean human landing captures. Captures conducted the same night, in the same area (Carbonnière vs Marais du Vigueirat) and in pixels of the same adult abundance index class were compared (the following adult abundance thresholds were chosen in order to determine the classes: 10; 100; 500; 1000; 2000; 5000 (Table 3 and 4). For example, human landing captures carried out on the $10^{\text {th }}$ of August in Carbonnière in pixel Hu5 and Hu6 were compared to light trap captures carried out on the same date and in the same area, in pixel LT1, LT4 and LT5 (all of these pixels have an adult abundance index between 100 and 500). Comparison showed a highly significant linear relationship between CDC-light traps and human captures $\left(\mathrm{r}^{2}=0.66, \mathrm{p}<0.01\right)$. Indeed, human biting rate $(m a)$ was extrapolated from the following formula: $m a=$ $(0.39 \mathrm{LT}+0.049) * 1.25$. As human landing captures refer to the hour following sunset, a correction factor of 1.25

Table 2: Total number of An. hyrcanus collected per month and associated percentages (bracket)

\begin{tabular}{|c|c|c|c|c|c|c|c|c|}
\hline & \multicolumn{8}{|c|}{ Capture places } \\
\hline & Méjannes & Aimargues & Mourgues & Pont de gau & Carbonnière & Vigueirat & $\begin{array}{l}\text { Western } \\
\text { Camargue }\end{array}$ & $\begin{array}{l}\text { Tour du } \\
\text { Vallat }\end{array}$ \\
\hline Year & 2005 & 2005 & 2005 & 2005 & 2005 & 2005 & 1969-1994 & 2004 \\
\hline $\begin{array}{l}\text { Capture } \\
\text { method }\end{array}$ & $\mathrm{CO} 2$ traps & $\mathrm{CO} 2$ traps & $\mathrm{CO} 2$ traps & $\mathrm{CO} 2$ traps & $\begin{array}{c}\text { CDC-LT + } \\
\text { CO2 }\end{array}$ & $\begin{array}{c}\text { CDC-LT + } \\
\text { CO2 }\end{array}$ & $\begin{array}{l}\text { Human landing } \\
\text { catch }\end{array}$ & $\begin{array}{l}\text { Horse bait } \\
\text { trap }\end{array}$ \\
\hline Reference & $\begin{array}{l}\text { G. L'Ambert, } \\
\text { unpublished } \\
\text { data }\end{array}$ & $\begin{array}{c}\text { G. L'Ambert, } \\
\text { unpublished } \\
\text { data }\end{array}$ & $\begin{array}{l}\text { A. Carron, } \\
\text { unpublished } \\
\text { data }\end{array}$ & $\begin{array}{l}\text { A. Carron, } \\
\text { unpublished } \\
\text { data }\end{array}$ & [4] & [4] & $\begin{array}{c}\text { EID- } \\
\text { Méditérannée, } \\
\text { unpublished data }{ }^{2}\end{array}$ & [3] \\
\hline March & - & - & - & - & $0(0)$ & $5(0)$ & $(0)$ & - \\
\hline April & - & - & - & - & $0(0)$ & $7(0)$ & (0) & - \\
\hline May & I (0) & $0(0)$ & $2(1)$ & $2(0)$ & $16(0)$ & $296(0)$ & (0) & $0(0)$ \\
\hline June & $27(0)$ & $0(0)$ & $18(7)$ & $28(3)$ & $673(12)$ & $6737(6)$ & (4) & $3(0)$ \\
\hline July & $357(5)$ & $0(0)$ & $104(42)$ & $107(12)$ & $374(7)$ & $17739(16)$ & (II) & $38(1)$ \\
\hline August & $5933(83)$ & $102(95)$ & $94(38)$ & $730(84)$ & 3257 (59) & $61315(55)$ & (43) & 4101 (7I) \\
\hline September & $857(12)$ & $5(5)$ & $32(13)$ & $7(1)$ & $1228(22)$ & $25708(23)$ & (42) & $1640(28)$ \\
\hline October & $0(0)$ & $0(0)$ & $0(0)$ & $0(0)$ & $3(0)$ & $124(0)$ & (0) & $4(0)$ \\
\hline Total & 7175 & 107 & 250 & 874 & 5551 & 111919 & & 5786 \\
\hline
\end{tabular}

Note:

I approximatively 12 points

2 mean percentage 
Table 3: Number of An. hyrcanus captured during human landing catches sessions.

\begin{tabular}{|c|c|c|c|c|c|c|c|c|c|}
\hline Area & Pixel & Adult abundance index & $08 / 08 / 2005$ & $10 / 08 / 2005$ & $19 / 09 / 2005$ & $21 / 09 / 2005$ & $04 / 10 / 2005$ & $23 / 08 / 2006$ & $05 / 09 / 2006$ \\
\hline Vigueirat & $\mathrm{Hul}$ & 8676 & 6312 & - & 0 & - & 14 & - & - \\
\hline Vigueirat & $\mathrm{Hu} 2$ & 122 & 26 & - & 0 & - & 0 & - & - \\
\hline Vigueirat & Hu3 & 308 & 2 & - & 9 & - & 0 & - & - \\
\hline Vigueirat & $\mathrm{Hu} 4$ & 2430 & - & - & - & - & - & - & 67 \\
\hline Carbonnière & Hu5 & 242 & - & 304 & - & 25 & - & - & - \\
\hline Carbonnière & Hu6 & 155 & - & 2 & - & 1 & - & - & - \\
\hline Carbonnière & Hu6 & 155 & - & 0 & - & 0 & - & - & - \\
\hline Carbonnière & Hu8 & 687 & - & - & - & - & - & 39 & - \\
\hline Carbonnière & Hu5 & 242 & - & - & - & - & - & 156 & - \\
\hline Carbonnière & $\mathrm{Hu} 7$ & 5348 & - & - & - & - & - & 16 & - \\
\hline Carbonnière & $\mathrm{Hu} 7$ & 5348 & - & - & - & - & - & 4 & - \\
\hline
\end{tabular}

Adult abundance index relative to the pixel where the capture was conducted is indicated. Human landing results are relative to the hour following sunset which represent the An. hyrcanus aggressiveness peak [4] and approximately $80 \%$ of the total number of An. hyrcanus captured during the entire night. Each line corresponds to a human landing catch session carried out by one volunteer belonging to the research team (two people were present at $\mathrm{Hu} 6$ and Hu7).

was associated with the estimation of $m a$ in order to obtain $m a$ for the entire night (Table 3).

The result is the spatio-temporal distribution of $m a$ in the Camargue, i.e., an estimation of the human biting rate for each $30 \mathrm{~m} \times 30 \mathrm{~m}$ pixel and each month. The human biting rate was figured for the month of August in order to illustrate this paragraph (Figure 4). Variability and uncertainty were taken into account for each step leading to the assessment of variability and uncertainty of $m a$ for each pixel.

Anopheles hyrcanus anthropophily (h)

(Figure 2) was estimated from the comparison between human landing, light traps and horse bait trap results for
An. hyrcanus and other Anopheles species [4], and was fitted as follows: Pert distribution $(0.4 ; 0.5 ; 0.8)$ (Figure 5). It was assumed that $h$ did not vary throughout the year.

The length of the trophogonic cycle (u)

(Figure 2) was calculated using the following formula, which estimates the length of time of blood digestion: $u=$ $f_{1} /\left(T-g_{1}\right)$ where $f_{1}$ and $g_{1}$ are factors depending on humidity and $T$ is the temperature. $f_{1}$ and $g_{1}$ were experimentally determined and evaluated at $36.5^{\circ} \mathrm{C}$-days and $9.9^{\circ} \mathrm{C}$, respectively, when the humidity reached $70-80 \%$ (from June to September 2005, mean monthly humidity varied from 63 to 90\% in the Camargue) [23]. Moreover, Detinova assumed that it was reliable to add 24 hours to take into account the time necessary to find a host and the time

Table 4: Number of An. hyrcanus captured during light trap sessions. Adult abundance index relative to the pixel where the capture was conducted is indicated.

\begin{tabular}{|c|c|c|c|c|c|c|c|c|c|}
\hline Area & Pixel & Adult abundance index & 08/08/2005 & $10 / 08 / 2005$ & $19 / 09 / 2005$ & $04 / 10 / 2005$ & $23 / 08 / 2006$ & 05/09/2006 & $21 / 09 / 2005$ \\
\hline Vigueirat & LTI & 346 & 218 & - & 346 & 6 & - & - & - \\
\hline Vigueirat & $\mathrm{LT} 2$ & 208 & 70 & - & 208 & I & - & - & - \\
\hline Vigueirat & LT3 & 6594 & 3546 & - & 6594 & 20 & - & - & - \\
\hline Vigueirat & LT4 & 10920 & |597| & - & 10920 & 15 & - & - & - \\
\hline Vigueirat & LT5 & 4898 & 2016 & - & 4898 & 32 & - & - & - \\
\hline Vigueirat & LT6 & 1769 & 180 & - & 1769 & 3 & - & 1200 & - \\
\hline Vigueirat & LT7 & 3732 & 3645 & - & 3732 & 4 & - & - & - \\
\hline Vigueirat & LT8 & 2681 & 1006 & - & 2681 & 24 & - & - & - \\
\hline Carbonnière & LTI & 492 & - & 254 & - & - & - & - & 8 \\
\hline Carbonnière & $\mathrm{LT} 2$ & 1299 & - & 102 & - & - & 101 & - & 10 \\
\hline Carbonnière & LT3 & 1250 & - & 39 & - & - & - & - & 0 \\
\hline Carbonnière & LT4 & 433 & - & 20 & - & - & - & - & 3 \\
\hline Carbonnière & LT5 & 176 & - & 288 & - & - & - & - & 3 \\
\hline Carbonnière & LT6 & 705 & - & 30 & - & - & 78 & - & 19 \\
\hline Carbonnière & LT7 & 721 & - & 17 & - & - & - & - & 15 \\
\hline Carbonnière & LT8 & 0 & - & 1 & - & - & - & - & 0 \\
\hline Carbonnière & LT9 & 5348 & - & - & - & - & 28 & - & - \\
\hline
\end{tabular}

Light trap captures are relative to the entire night. 


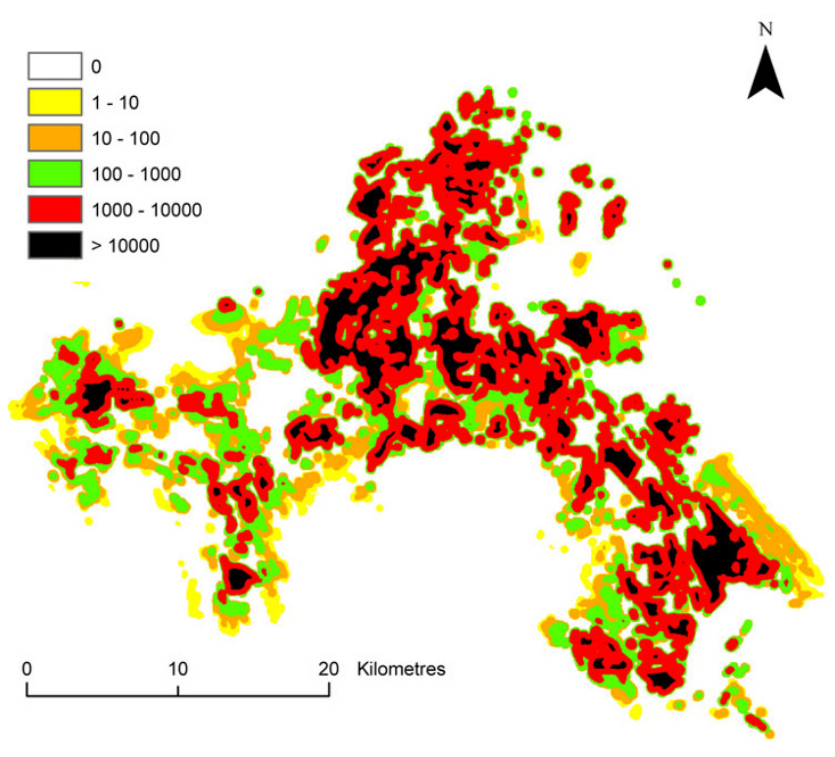

Figure 4

Spatial distribution of the human biting rate in the Camargue in August. Classes were arbitrary chosen with a logarithmic scale.

necessary to find a pool to lay eggs in, in order to fulfil a complete gonotrophic cycle [23]. Considering expert knowledge and bibliographical data [24], a two day range of values was fixed for each month in the Camargue, and the length of the trophogonic cycle was fitted as indicated in Table 5.

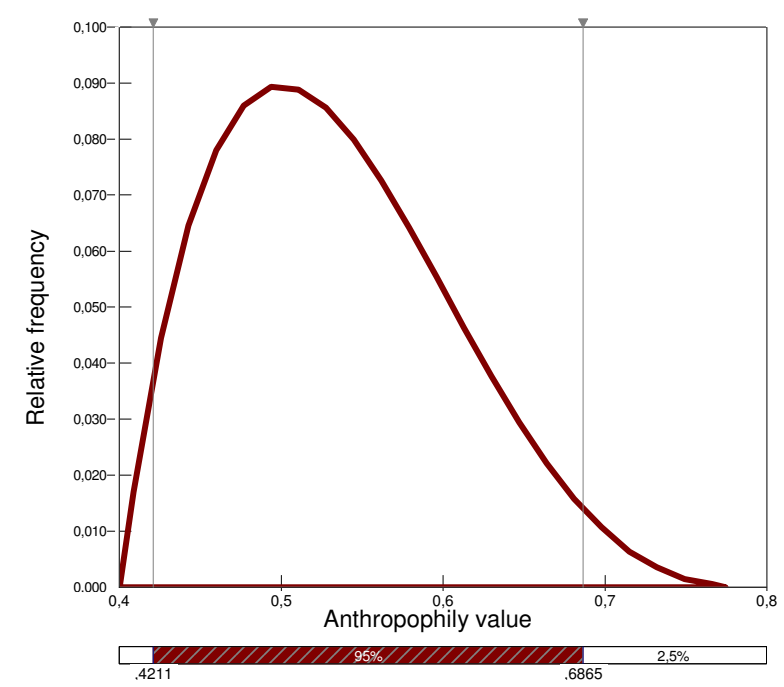

Figure 5

Distribution of An. hyrcanus anthropophily.
In order to illustrate this, the distribution of the length of the trophogonic cycle was determined for August (Figure 6).

The daily survival rate $(\mathrm{P})$

(Figure 2) was estimated from parity rates (P) using the following formula: $p=\mathrm{P}^{1 / \mathrm{u}}[25]$ which is relevant in the case of stable populations. Considering that the An. hyrcanus population increases and decreases progressively according to the Anopheles biology, the approximation that populations were stable during the summer was used [5]. Parity rates observed in June, July and September 2005 in Carbonnière and Marais du Vigueirat were used (due to the low number of dissected mosquitoes, the parity rate calculated in June in Carbonnière was not included) [4]. They were fitted as indicated in Table 6, and the mean value was calculated from the five probability distributions in order to obtain the mean An. hyrcanus parity rate in the Camargue from June to September 2005.

During the same period (June to September 2005), the daily temperature varied from $18.5^{\circ} \mathrm{C}$ to $26.4^{\circ} \mathrm{C}$, with a mean value of $23.2^{\circ} \mathrm{C}$. The length of the trophogonic cycle, calculated using the same method as before, ranged from 3.2 days to 5.2 days, with a mean value of 3.7 , and was fitted as follows: Pert distribution $(3 ; 3.5 ; 5.5)$. Using the formula $p=\mathrm{P}^{1 / \mathrm{u}}$, a mean $p$ value of 0.79 was obtained (Figure 7). Due to difficulty in obtaining a precise value for parity rates for the entire year, it was considered that $p$ did not vary from March to October in this model.

The length of the sporogonic period (n)

(Figure 2) was calculated as follows: $n=f_{2} /\left(T-g_{2}\right)$, where $T$ is temperature and $f_{2}$ and $g_{2}$ are Plasmodium speciesdependent factors [26]. $f_{2}$ and $g_{2}$ were experimentally evaluated at $111^{\circ} \mathrm{C}$-days and $16^{\circ} \mathrm{C}, 105^{\circ} \mathrm{C}$-days and $14.5^{\circ} \mathrm{C}$ for Plasmodium falciparum and P. vivax, respectively. However, Grassi and MacDonald estimated threshold temperatures under which the sporogonic development is not completed: $18-19^{\circ} \mathrm{C}$ for $P$. falciparum, $15-17^{\circ} \mathrm{C}$ for $P$. vivax $[24,27]$.

\section{Entomological data: infectivity}

Anopheles hyrcanus susceptibility was estimated based on data derived from experimental membrane-feeding experiments conducted with cultured $P$. falciparum in the laboratories of Radboud University Nijmegen Medical Centre (The Netherlands) following a routine protocol $[28,29]$. Among 350 An. hyrcanus tested, none were found able to transmit tropical P. falciparum strains. Hence, An. hyrcanus susceptibility to $P$. falciparum was fitted with a beta distribution: Beta $(1 ; 351)$ (Figure 8$)$.

Anopheles hyrcanus susceptibility to $P$. vivax has not yet been tested, but this species has been considered a vector 
Table 5: Length of the trophogonic cycle

\begin{tabular}{cccc}
\hline Month & Mean temperature (2002-2006) & Length of trophogonic cycle (from Shlenova and Detinova) & Pert distribution \\
\hline March & 10.9 & 38.4 & $(9 ; 10 ; 11)$ \\
April & 14 & 9.9 & $(4.5 ; 5.5 ; 6.5)$ \\
May & 17.8 & 5.6 & $(3 ; 4 ; 5)$ \\
June & 23 & 3.8 & $(3 ; 3.5 ; 5)$ \\
July & 24.8 & 3.4 & $(3 ; 3.5 ; 5)$ \\
August & 24 & 3.6 & $(3.5 ; 4.5 ; 5.5)$ \\
September & 20.3 & 4.5 & $(5 ; 6 ; 7)$ \\
October & 17.1 & 6.1 & \\
\hline
\end{tabular}

of $P$. vivax in Afghanistan for more than 30 years [30,31]. Genetic comparison based on ITS2 sequences between French, Afghan, Turkish and Iranian specimens concluded that they were identical. Hence, it was considered that $A n$. hyrcanus was susceptible to tropical $P$. vivax strains, and estimation of its susceptibility, based on expert knowledge, was fitted as follows: Pert distribution $(0.05 ; 0.20$; 0.70) (Figure 9).

An. hyrcanus susceptibility to either $P$. falciparum or $P$. vivax was considered to be homogeneous through time and space (Table 1).

Anopheles hyrcanus susceptibility to Plasmodium ovale and Plasmodium malariae was not inferred, as very little is known about European Anopheles susceptibility to these two species.

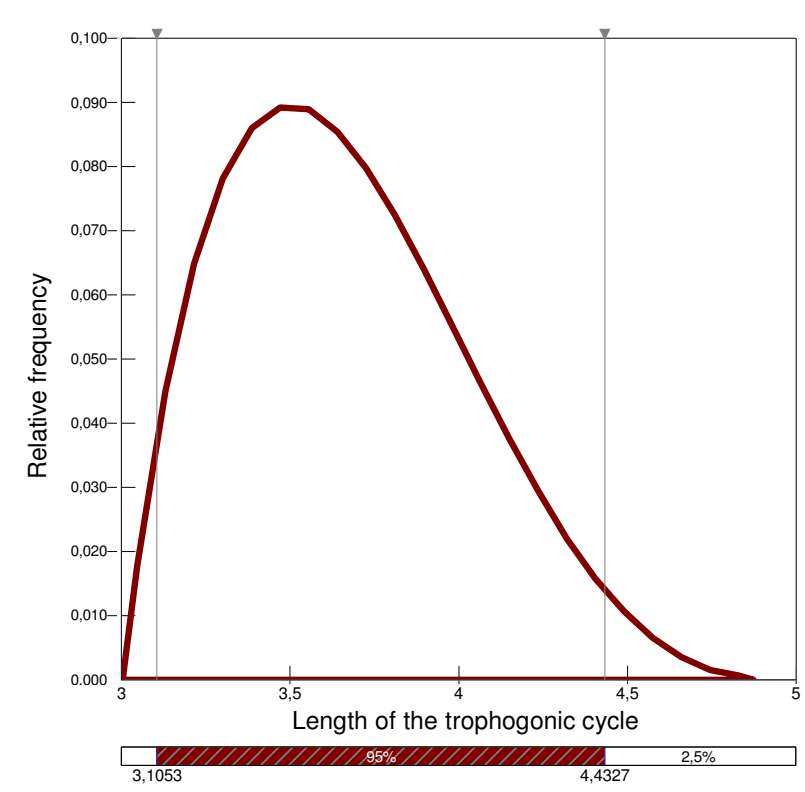

Figure 6

Distribution of the length, in days, of the trophogonic cycle in August.

\section{Parasitological data: vulnerability}

Vulnerability is related to gametocyte carriers as the gametocyte is the stage transmissible to mosquitoes. Nevertheless, very little is known about gametocyte carriers, and any information must be considered in the context of imported malaria cases, i.e. taking account of the fact that the illness is diagnosed and treated. Since anti malarialtreatment in France is generally conducted with drugs that do not prevent gametocyte emergence [8], an assumption that every patient may develop some gametocytes was made. Hence, we used imported malaria cases to approach the gametocyte presence in the Camargue.

Data relative to imported malaria cases were obtained from five public hospitals localized in important towns in/around the Camargue (Montpellier, Nîmes, Avignon, Arles and Marseille). These data were analysed with regard to the date, Plasmodium species, patients' residence for the 2004-2005 period and contamination place.

It was estimated that imported malaria case data obtained from public hospitals represented about $50-55 \%$ of all imported malaria cases, with other cases being diagnosed by private laboratories $[32,33]$. Thus, the total number of imported malaria cases was estimated using a $52.5 \%$ correcting factor assuming that epidemiological data provided by public hospitals were representative of the total number of imported malaria cases.

\section{Results \\ Vulnerability}

In 2004 and 2005, 657 imported cases were diagnosed in the region (corresponding to a total of 1251 estimated imported cases), among which $P$. falciparum, P. vivax, $P$.

Table 6: Parity rates observed in the Camargue in 2005

\begin{tabular}{ccc}
\hline & Carbonnière & Marais du Vigueirat \\
\hline June & - & Beta $(18 ; 9)$ \\
July & Beta $(20 ; 69)$ & Beta $(29 ; 91)$ \\
September & Beta $(89 ; 45)$ & Beta $(40 ; 80)$ \\
\hline
\end{tabular}




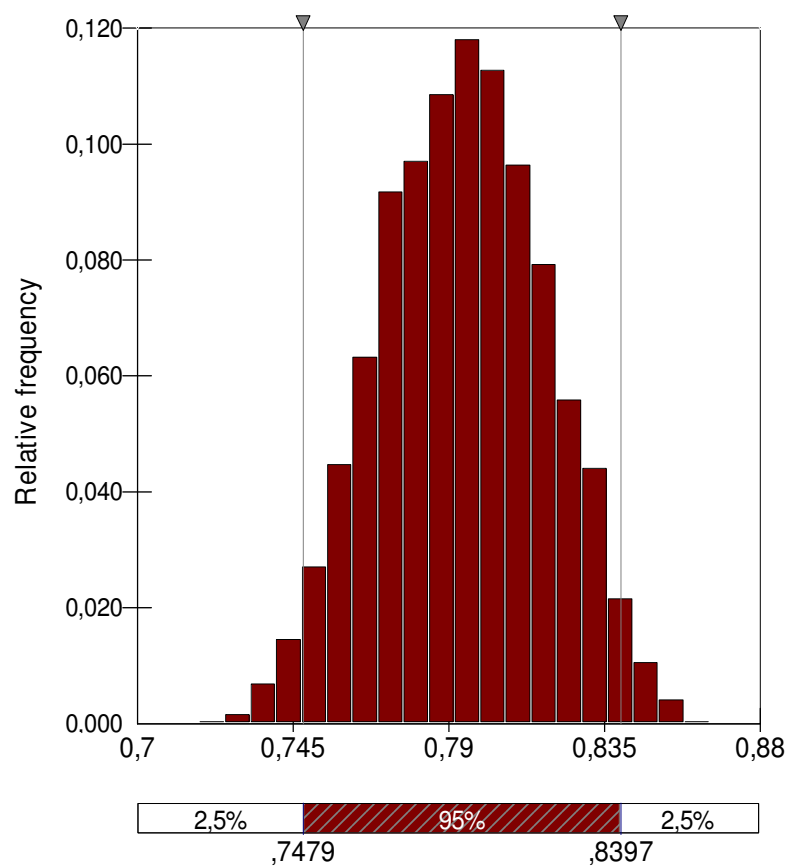

Figure 7

Distribution of the An. hyrcanus daily survival rate.

ovale and $P$. malariae represented $85.5 \%, 7 \%, 5.5 \%$ and $2 \%$, respectively. Moreover, $35 \%$ of these cases occurred between August and September. Among 528 patients for whom the place of residence was known, 75\% were living

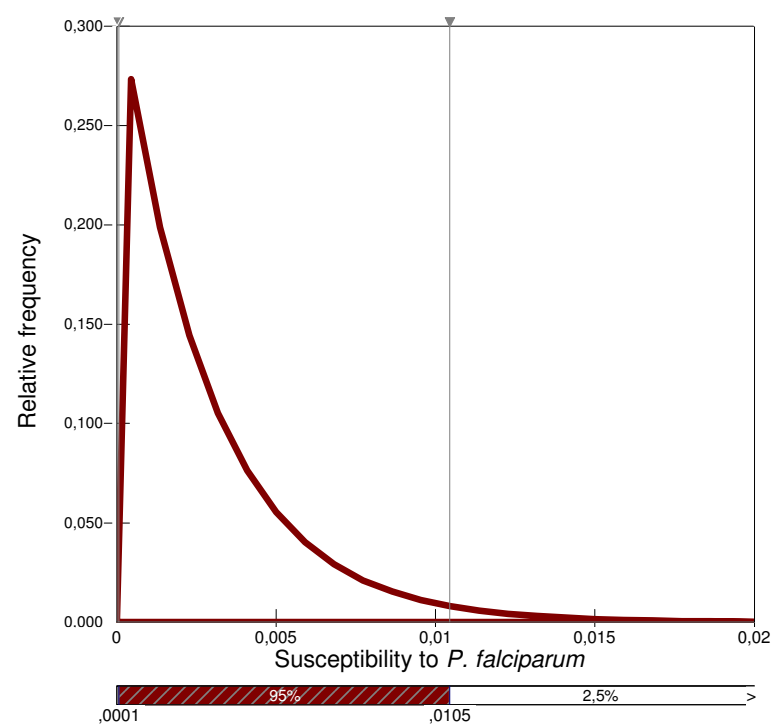

Figure 8

Distribution of An. hyrcanus susceptibility to $P$. falciparum.

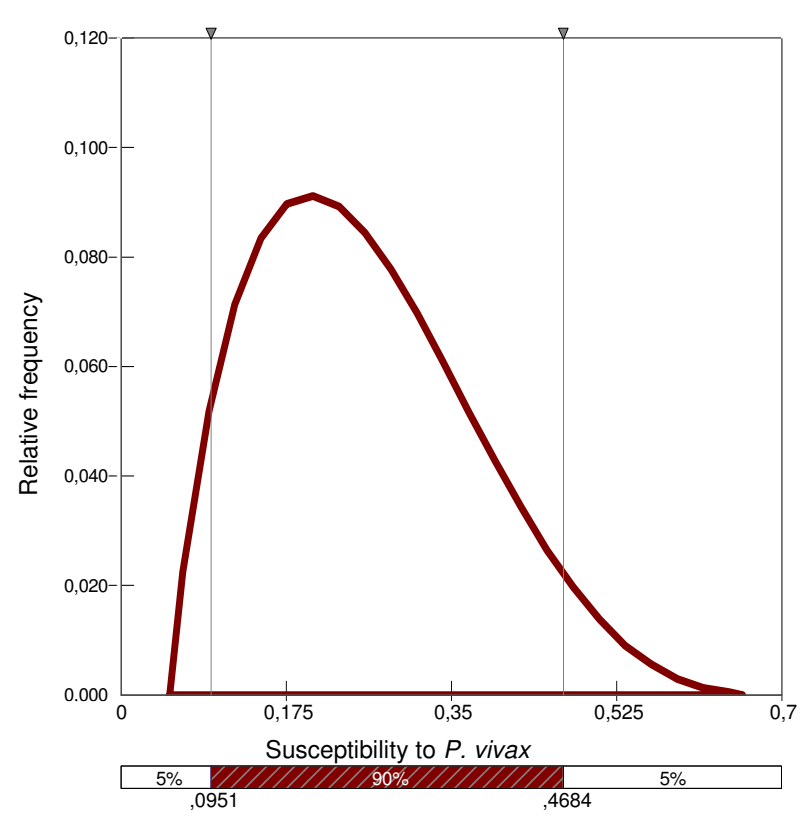

Figure 9

Distribution of An. hyrcanus susceptibility to $P$. vivax.

in Montpellier, Nîmes, or Marseille big cities, and only 7 patients were living in areas where An. hyrcanus could be present.

Countries in which patients were infected were not known for 2004-2005. For the 2001-2003 period, 96\% of imported malaria cases were contracted in Africa, and the Comoros Islands represented the main place of contamination for cases diagnosed in Marseille, Arles and Nîmes. Data collected in Montpellier and Avignon showed that imported cases were mainly contracted in West Africa.

\section{Entomological risk (receptivity*infectivity)}

The mean value of the entomological risk was assessed for $P$. falciparum species from June to September, and for $P$. vivax for the month of August for each pixel of the Camargue map (Figure 10, 11, 12, 13, 14) (the entomological risk was not assessed for $P$. ovale and $P$. malariae due to the low number of imported cases and the lack of information concerning both species). It was calculated with 10,000 different randomly selected sets of values extracted from input distributions.

Strong differences were observed in the entomological risk for the two Plasmodium species: P. falciparum transmission risk estimate ranges from 0 to more than 1 although P. vivax transmission risk estimate ranges from 0 to more than 100 . 


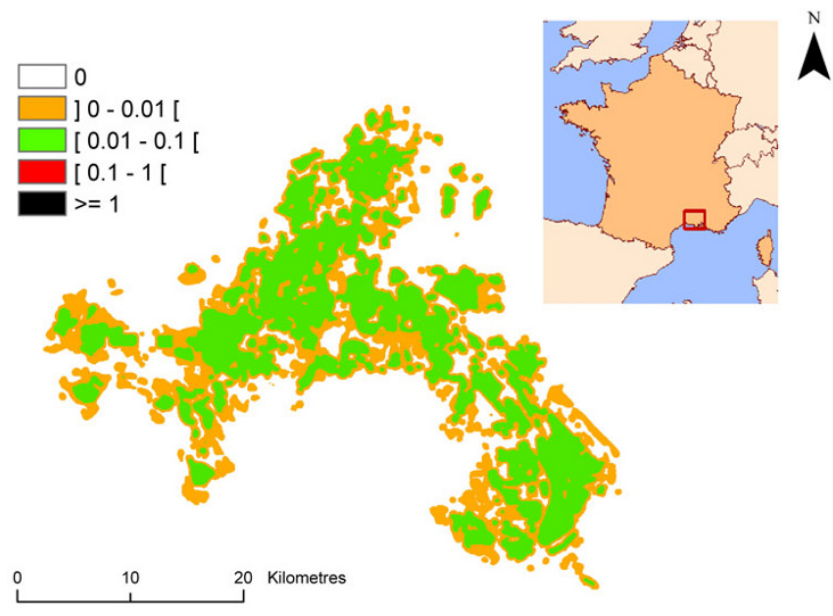

Figure 10

Spatial variations of $\boldsymbol{P}$. falciparum transmission risk estimate (ranging from $\mathbf{0}$ to more than I) in June in the Camargue. Classes were arbitrary chosen with a logarithmic scale.

\section{Uncertainties of the risk estimate and sensitivity analysis}

For each pixel the outcome of the model is a statistical distribution of the risk estimate, generated by variability and uncertainty within inputs. For example, the entomological risk estimate ranges from about $9.10^{-6}$ to 20 , with $95 \%$ of the values being between 0.016 and 4.7 for pixel having a mean value of about 1 .

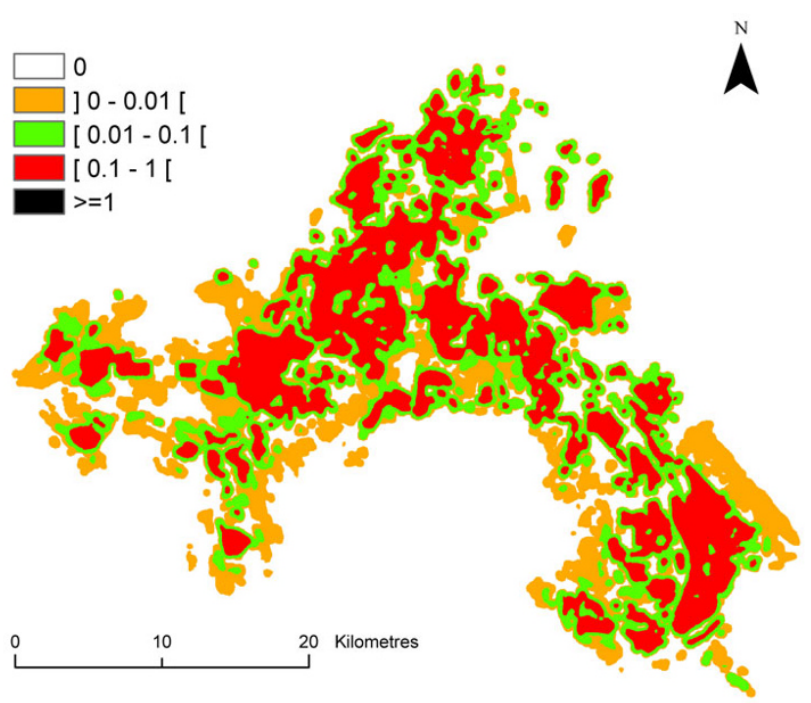

Figure I I

Spatial variations of $P$. falciparum transmission risk estimate (ranging from 0 to more than I) in July in the Camargue. Classes were arbitrary chosen with a logarithmic scale.

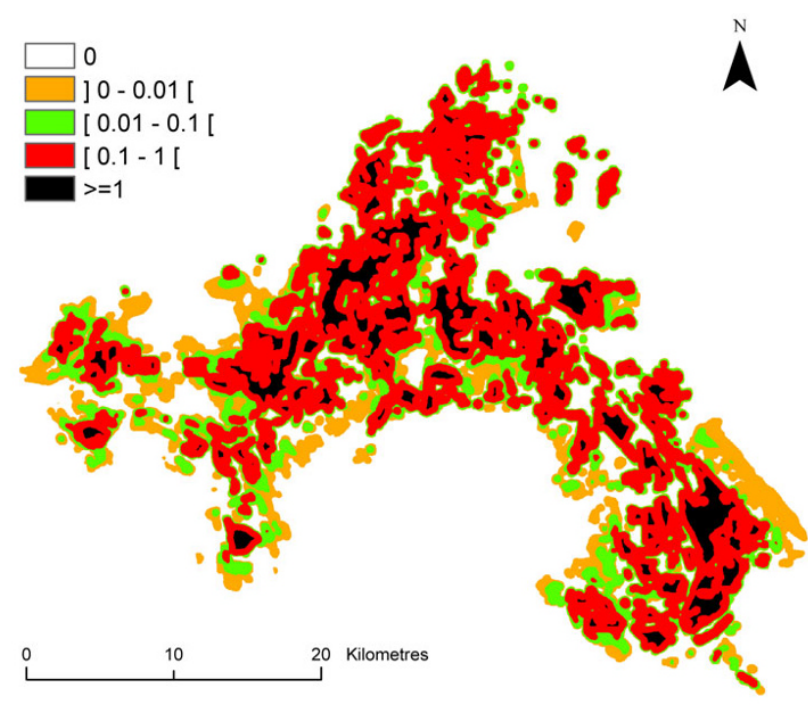

Figure 12

Spatial variations of $P$. falciparum transmission risk estimate (ranging from 0 to more than I) in August in the Camargue. Classes were arbitrary chosen with a logarithmic scale.

The sensitivity analysis was conducted for pixels of which the mean entomological risk estimate is about 1 . Results of the sensitivity analysis show the correlation coefficients

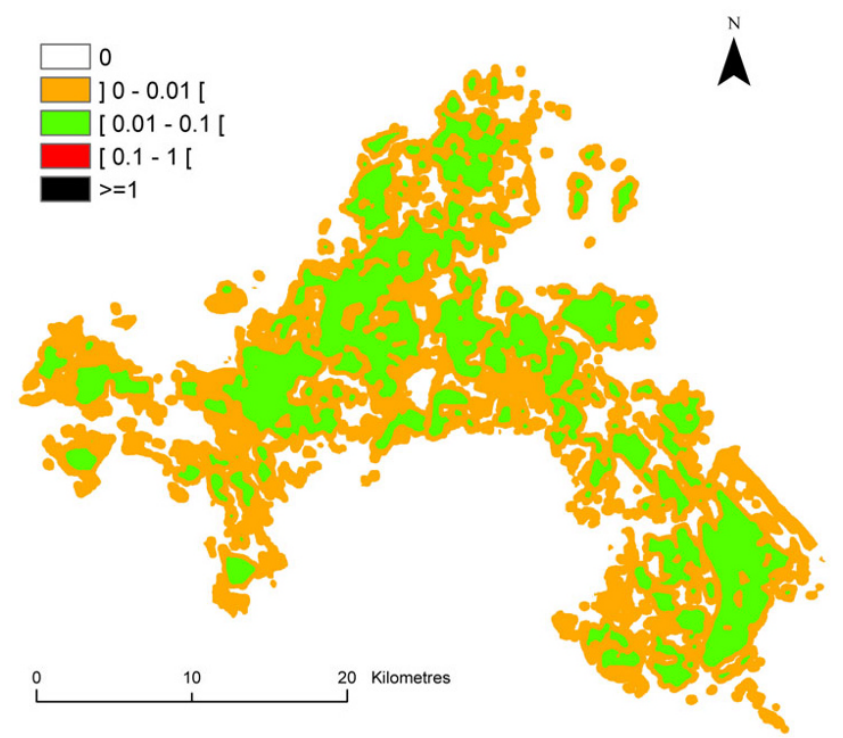

Figure 13

Spatial variations of $P$. falciparum transmission risk estimate (ranging from 0 to more than I) in September in the Camargue. Classes were arbitrary chosen with a logarithmic scale. 


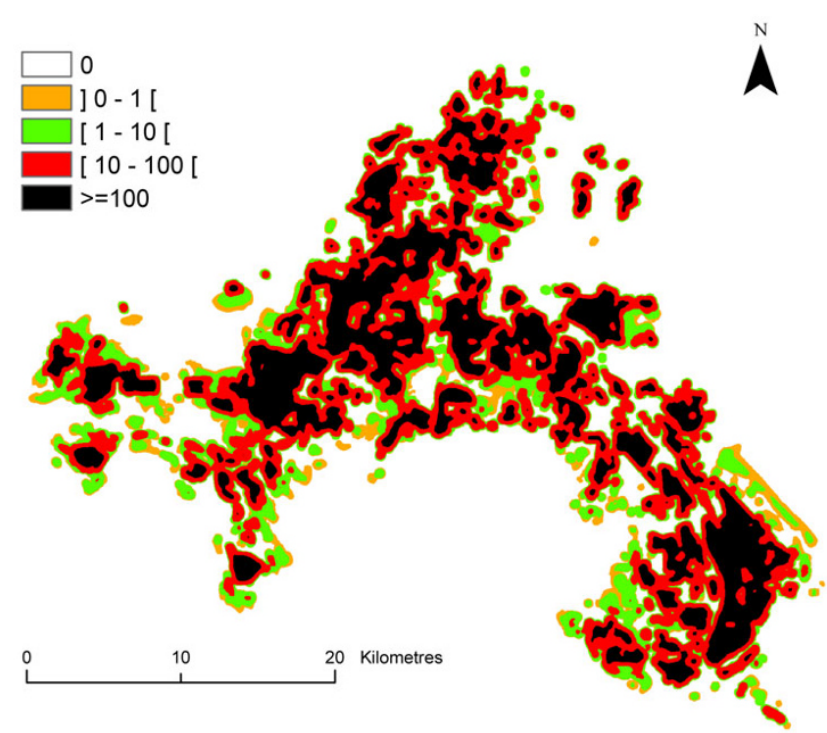

Figure I4

Spatial variations of the $P$. vivax transmission risk estimate (ranging from 0 to more than 100 ) in August in the Camargue. Classes were arbitrary chosen with a logarithmic scale.

between different varying inputs and the consecutive varying risk estimate (Figure 15).

Sensitivity analysis carried out for pixels presenting a lower risk estimate showed a predominance of susceptibility. However, correlation coefficients of the survival rate and the human biting rate were approximately equal. Sensitivity analysis carried out for the P. vivax entomological risk estimate showed the equal importance of the susceptibility, the survival rate and the human biting rate.

\section{Discussion}

The entomological risk index used in this article refers to the risk of transmission (Figure 1): the risk of transmission being high when the entomological risk is high. Results clearly indicate a maximum risk of $P$. falciparum transmission in August in the Camargue considering the spatial distribution and value of the entomological risk estimate, which results from length of the trophogonic cycle and sporogonic period and the human biting rate. The length of the trophogonic cycle and sporogonic development period are directly influenced by the mean temperature in our model, and the human biting rate depends directly on the An. hyrcanus dynamics and density [5]. The human biting rate being the only space-dependant factor in our model, one could be tempted to approach roughly the risk of transmission by the human biting rate. Nevertheless, the entomological risk presents also temporal variations, which depend not only on the human biting rate

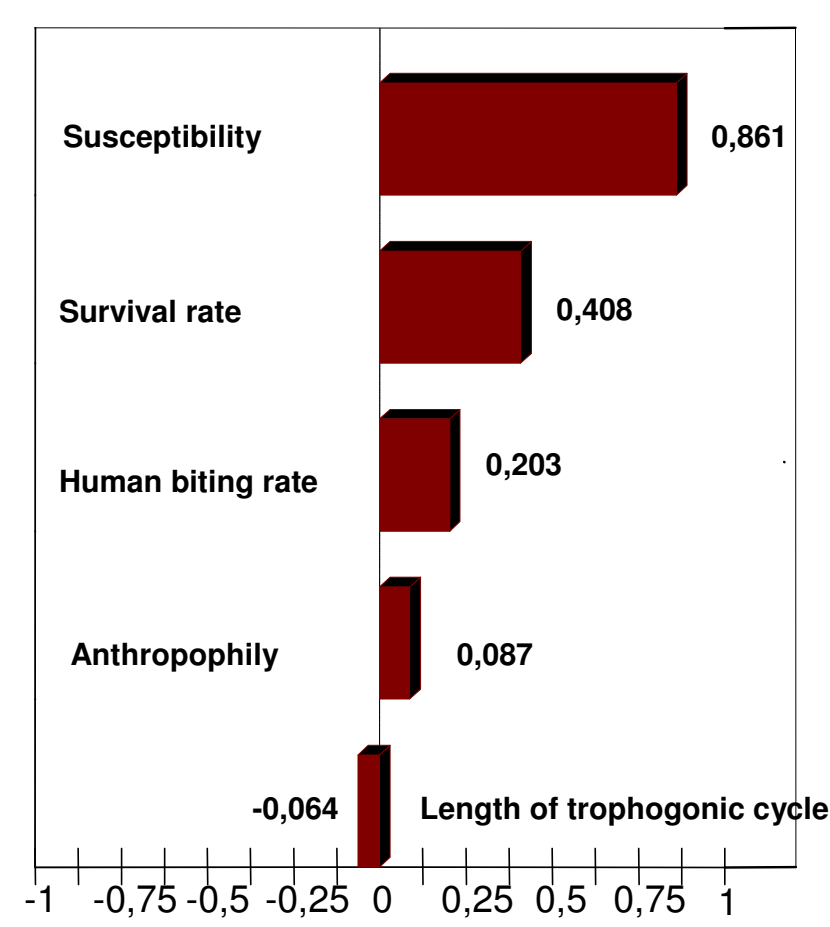

Correlation Coefficients

Figure I 5

Sensitivity analysis.

but also on the impact of temperature on the length of the trophogonic and sporogonic cycle. Dynamics of An. hyrcanus and mean temperature do not evolve exactly in the same way in the Camargue, which shows the necessity of estimating all the parameters of the entomological risk.

The risk of $P$. vivax transmission is more than one hundred times higher than the risk of $P$. falciparum transmission, which is due to infectivity and the length of sporogonic cycle differences.

Nevertheless, the entomological risk calculated herein is a theoretical index as the human biting rate reaches more than 10,000 bites per human per night reflecting the abundance of An. hyrcanus. This will, of course, never happen as no one can endure such a large number of bites. This suggests that, in the future, it could be necessary to combine the entomological risk with human presence and exposure to mosquito bites in order to evaluate the real human biting rate. Such analyses would require complementary geographical and sociological studies.

Although all of France faces a large number of imported cases [8], particularly in the south-east of the country, vul- 
nerability in at risk areas is very low because most imported cases are present in large cities.

The entomological risk, referring to the risk of transmission in this article (Figure 1), has to be interpreted in a relative way due to its definition and the estimation of its parameters. Thus, this study underlined space, time and Plasmodium species-dependant of the risk of potential transmission. Of course, the risk of potential transmission is connected with the risk of malaria re-emergence when gametocytes carriers are introduced within at risk areas. Considering the entomological risk and the length in days of the infectious period of humans would allow estimation of R0 and the following absolute risk of malaria reemergence. Finally, the current risk of malaria re-emergence seems negligible due to the very low number of imported Plasmodium.

As stated in the introduction, the aim of this study was not to build a public health tool for controlling malaria in the Camargue. The emphasis was on presenting an innovative approach of spatialized quantitative risk assessment applied to a vector-borne disease, which has not been previously conducted. The main advantages of such a probabilistic approach are the possibility of integrating the uncertainty and variability of inputs within a model and to quantify the uncertainty of the final risk estimate. The deterministic approaches used thus far have not taken uncertainty and variability into account [27,34-36] and have produced precise outcomes, which could lead to misinterpretation as the final risk estimate could vary significantly due to input variability. Integration of uncertainty and variability in deterministic models would rapidly lead to complicated models, requiring laborious mathematical developments [18].

Moreover, the approach applied in this study is based on distributions that are combined, resulting in quite sophisticated analyses that are intuitive and easily understood. The uncertainty within the risk estimate is a crucial point for decision makers, which usually apply some rough "safety margin" around a deterministic estimate to express their feeling of uncertainty. The pros of the approach developped in this article are rightly to quantify this uncertainty.

Such a method could be applied to other areas where malaria is still a threat or to emerging vector-borne diseases, such as the dengue or chikungunya virus infections. This method could be used in a controlled way, in order to identify areas and time periods that correspond to the highest risk of transmission, and to focus control measures where and when transmission is elevated.
It has been shown recently the impact of anthropogenic changes on potential malaria vectors in the Camargue over the last 60 years [37]. This method could be used also to predict the probable impact of future decisions concerning land use for example, and could be a useful tool for decision makers.

The sensitivity analysis underlined factors responsible for entomological risk uncertainty and variation, of which susceptibility, the survival rate and the human biting rate of An. hyrcanus have a major impact in this model. Variability in either of these parameters leads to variability and uncertainty in the risk estimate. In contrast to the susceptibility, survival rate and human biting rate, variability in the anthropophily range and the length of the trophogonic cycle range has only a minor impact on the risk estimate. The sensitivity analysis thus appears to be a tool particularly useful for the identification of key factors, which need to be assessed in field surveys.

In the context of emerging vector-borne diseases, emphasis is currently on developing and improving such quantitative risk assessment models integrating variability and uncertainty of biological parameters, which are usually difficult to assess, and especially parameters recorded in the field.

\section{Competing interests}

The authors declare that they have no competing interests.

\section{Authors' contributions}

NP designed the study, analysed the data and drafted the manuscript. AT analysed the data and carried out spatial analysis. CT participated in data collection and analysis. $\mathrm{AL}$ managed the susceptibility trials. DF conceived of the study, participated in its design and coordination and helped to draft the manuscript. All authors read and approved of the final manuscript.

\section{Acknowledgements}

We thank Geert-Jan van Gemert and Marga van de Vegte-Bolmer for technical expertise in conduct of the susceptibility trials. We thank Grégory L'Ambert, Jean Baptiste Ferré, Charles Jeannin, Claire Duchet and Alexandre Carron from EID-Méditerranée for help in human landing captures and data on An. hyrcanus dynamics. We thank Philippe Parola and Marie Pierre Ranjeva from the North hospital of Marseille, Didier Basset from the hospital of Montpellier, Laurence Lachaud from the hospital of Nîmes, Muriel Roumier from the hospital of Arles, Martine Derré from the hospital of Avignon, Anzime Ali Mohamed from the "Hôpital d'Instruction des Armées Laveran" and Frabrice Legros from the CNREPIA for providing data on imported malaria cases.

This work was partially funded by the French Ministry of Agriculture and EU grant GOCE-2003-0 I 0284 EDEN. The paper is catalogued by the EDEN Steering Committee as EDEN0106 http://www.eden-fp6project.net/. The contents of this publication are the sole responsibility of the authors and can in no way be taken to reflect the views of the European Union. 


\section{References}

I. Rodhain F, Charmot G: Evaluation des risques de reprise de transmission du paludisme en France. Med Mal Infect 1982, I 2(4):23I-236.

2. Sautet J: A propos d'une épidémie de paludisme en Camargue. Mars Med 1944, 2( I 5):53-64.

3. Balenghien T, Fouque F, Sabatier P, Bicout DJ: Horse, bird, and human-seeking behavior and seasonal abundance of mosquitoes in a West Nile virus focus of southern France. J Med Ento$\mathrm{mol} 2006$, 43(5):936-946.

4. Ponçon N, Toty C, L'Ambert G, Le Goff G, Brengues C, Schaffner F, Fontenille $\mathrm{D}$ : Biology and dynamics of potential malaria vectors in southern France. Malar J 2007, 6(I):18.

5. Ponçon N, Toty C, L'Ambert G, Le Goff G, Brengues C, Schaffner F, Fontenille $D$ : Population dynamics of pest mosquitoes and potential malaria and West Nile virus vectors in relation to climatic factors and human activities in the Camargue, France. Med Vet Entomol 2007, 2 1:350-357.

6. Ponçon N, Toty C, Kengne P, Alten B, Fontenille D: Molecular evidence for similarity between Anopheles hyrcanus (Diptera: Culicidae) and Anopheles pseudopictus (Diptera: Culicidae), sympatric potential vectors of malaria in France. J Med Entomol 2008, 45:576-580.

7. Doudier B, Bogreau H, DeVries A, Ponçon N, Stauffer WM, Fontenille D, Rogier C, Parola P: Autochthonous malaria from Marseille to Minneapolis? Emerg Infect Dis 2007, I3(8): I 236-I 238.

8. Legros F, Arnaud A, El Minouni B, Danis M: Paludisme d'importation en France métropolitaine : données épidémiologiques 2001-2004. Bulletin épidémiologique hebdomadaire 2006, 32:235-236.

9. WHO-CISID: WHO - CISID-malaria. [http://data.euro.who.int/ cisid/].

10. Ejov M: Imported malaria and the risk of malaria reintroduction in Europe. CD News, Quaterly Communicable Disease Report, WHO Europe 2004, 34:3-7.

II. Garrett-Jones C, Shidrawi GR: Malaria vectorial capacity of a population of Anopheles gambiae: an exercise in epidemiological entomology. Bull World Health Organ I969, 40(4):53 I-545.

12. WHO: Expert Committee on Malaria, Twelfth Report. Technical Report Series, $N^{\circ} 324$ 1966, 324:.

13. Garrett-Jones C: Prognosis for interruption of malaria transmission through assessment of the mosquito's vectorial capacity. Nature 1964, 204: I I73-I I75.

14. WHO: Receptivity to malaria and other parasitic diseases. In Euro Reports and Studies, 15 Copenhagen ; 1979:108.

15. Dervieux A, Allard P, Naizot T, Auda Y: La Camargue est-elle un polder? Changement et stabilité dans le delta du Rhône depuis le début du XIXème siècle. In Equilibre et ruptures dans les écosystèmes durant les 20 derniers millénaires en Europe de l'Ouest Volume I. Edited by: Richard H, Vignot A. Besançon ; 2002:3 I5-329.

16. Mathevet R, Mouret JC, Mesléard F: Du mondial au local : originalité et dépendances de la riziculture camarguaise. Faire savoirs 2002, 2:49-56.

17. Pouillot R, Miconnet N, Afchain AL, Delignette-Muller ML, Beaufort A, Rosso L, Denis JB, Cornu M: Quantitative risk assessment of Listeria monocytogenes in French cold-smoked salmon: I. quantitative exposure assessment. Risk analysis 2007, 27(3):683-700.

18. Vose DJ: The application of quantitative risk assessment to microbial food safety. J Food Prot 1998, 6 I(5):640-648.

19. Vose DJ: Risk analysis. A quantitative guide. Chichester, England , John Wiley and Sons, LTD; 2000.

20. Anderson EL, Hattis D: When and how can you specify a probability distribution when you don't know much. Foudations: A. Uncertainty and variability. Risk analysis 1999, 19:47-49.

21. Cassin MH, Lammerding AM, Todd EC, Ross W, McColl RS: Quantitative risk assessment for Escherichia coli O157:H7 in ground beef hamburgers. Int J Food Microbiol 1998, 4 I (I):2 I-44.

22. Tran A, Ponçon N, Toty C, Linard C, Guis H, Ferré JB, D.L. S, Roger $F$, de la Rocque $S$, Fontenille $D$, Baldet $T$ : Using remote sensing to map larval and adult populations of Anopheles hyrcanus (Diptera: Culicidae) potential malaria vector in Southern France. Int J Health Geogr 2008, 7:9.

23. Detinova TS: Age-grouping methods in Diptera of medical importance with special reference to some vectors of malaria. Monogr Ser World Health Organ 1962, 47:13-191.
24. Jetten TH, Takken W: Anophelism without malaria in Europe. A review of the ecology and distribution of the genus Anopheles in Europe. Wageningen, The Netherlands, Landbouwuniversiteit Wageningen (Wageningen Agricultural University); 1994:No. 94-5, 69.

25. Davidson G: Estimation of the survival rate of anopheline mosquitoes in nature. Nature 1954, I 74(4434):792-793.

26. Moshkovsky S, Rashina $M$ : Epidémiologie et parasitologie médicale à l'usage des entomologistes. Moscow ; I95I.

27. Macdonald G: The epidemiology and control of malaria. London , Oxford University Press; 1957:201.

28. Ponnudurai T, Verhave JP, Meuwissen JH: Mosquito transmission of cultured Plasmodium falciparum. Trans $\mathrm{R}$ Soc Trop Med Hyg 1982, 76(2):278-279.

29. Ponnudurai T, Billingsley PF, Rudin W: Differential infectivity of Plasmodium for mosquitoes. Parasitol Today 1988, 4(I I):3 I 9-32 I.

30. Onori E, Nushin MK, Cullen JE, Yakubi GH, Mohammed K, Christal FA: An epidemiological assessment of the residual effect of DDT on Anopheles hyrcanus sl and An. pulcherrimus (Theobald) in the North Eastern region of Afghanistan. Trans $R$ Soc Trop Med Hyg 1975, 69(2):236-242.

3I. Faulde MK, Hoffmann R, Fazilat KM, Hoerauft A: Malaria reemergence in Northern Afghanistan. Emerg Infect Dis 2007, I3(9): | 402-| 404

32. Legros F, Fromage M, Ancelle T, Burg E, Janot C, Maisonneuve P, Danis $M$ : Enquête nationale de recensement des cas de paludisme d'importation en France métropolitaine pour l'année 1997. Bulletin épidémiologique hebdomadaire 1999, I I:41-42.

33. Danis $M$, Legros $F$, Thellier M, Caumes E: Données actuelles sur le paludisme en France métropolitaine. Med Trop (Mars) 2002, 62(3):2|4-2|8.

34. Koella JC: On the use of mathematical models of malaria transmission. Acta Trop 1991, 49(I): I-25.

35. Martens P, Kovats RS, Nijhof S, de Vries P, Livermore MTJ, Bradley DJ, Cox J, McMichael AJ: Climate change and future populations at risk of malaria. Glob Environ Change 1999, 9:S89-S107.

36. Lindsay SW, Thomas CJ: Global warning and risk of vivax malaria in Great Britain. Global change and human health 200I, 2(I):80-84.

37. Ponçon N, Balenghien T, Toty C, Ferré JB, Thomas C, Dervieux A, L'Ambert G, Schaffner F, Bardin O, Fontenille D: Effects of local anthropogenic changes on potential malaria vector Anopheles hyrcanus and West Nile virus vector Culex modestus, Camargue, France. Emerg Infect Dis 2007, I3:1810-1815.

Publish with Bio Med Central and every scientist can read your work free of charge

"BioMed Central will be the most significant development for disseminating the results of biomedical research in our lifetime. "

Sir Paul Nurse, Cancer Research UK

Your research papers will be:

- available free of charge to the entire biomedical community

- peer reviewed and published immediately upon acceptance

- cited in PubMed and archived on PubMed Central

- yours - you keep the copyright

Submit your manuscript here:

http://www.biomedcentral.com/info/publishing_adv.asp
BiolMedcentral 